\title{
Conformal classes of asymptotically flat, static vacuum data.
}

\author{
Helmut Friedrich \\ Max-Planck-Institut für Gravitationsphysik \\ Am Mühlenberg 1 \\ 14476 Golm, Germany
}

November 23, 2018

\begin{abstract}
We show that time-reflection symmetric, asymptotically flat, static vacuum data which admit a non-trivial conformal rescaling which leads again to such data must be axi-symmetric and admit a conformal Killing field. Moreover, it is shown that there exists a 3 -parameter family of such data.
\end{abstract}

PACS: 04.20.Ex, 04.20.Ha, 04.20.Jb

\section{Introduction}

The metric of a static space-time assumes in suitable local coordinates $t, \tilde{x}^{a}, a=1,2,3$, the form

$$
\tilde{g}=v^{2} d t^{2}+\tilde{h}, \quad v=v\left(\tilde{x}^{c}\right)>0, \quad \tilde{h}=\tilde{h}_{a b}\left(\tilde{x}^{c}\right) d \tilde{x}^{a} d \tilde{x}^{b},
$$

where $\tilde{h}$ denotes a negative definite metric on the time slices $\tilde{S}_{c}=\{t=c=$ const. $\}$. The hypersurface orthogonal, time-like Killing vector field is then given by $\partial_{t}$. We refer to $\tilde{h}$ as the static metric and to $v$ as the potential. Einstein's vacuum field equations reduce here to the static vacuum field equations

$$
R_{a b}[\tilde{h}]=\frac{1}{v} \tilde{D}_{a} \tilde{D}_{b} v, \quad \Delta_{\tilde{h}} v=0,
$$

where $\tilde{D}$ denotes the covariant derivative defined by $\tilde{h}$. It suffices to consider these equations on $\tilde{S} \equiv \tilde{S}_{0}$. 
In the following we study solutions which are asymptotically flat so that the coordinates $\tilde{x}^{a}$ can be required, for suitable $\tilde{S}$, to map the set $\tilde{S}$ diffeomorphically onto the complement of a closed ball $B_{R}(0)$ in $\mathbb{R}^{3}$ so that the fields $\tilde{h}, v$ satisfy with some $\epsilon>0$ and $k \geq 2$ the asymptotic flatness condition 1

$$
\tilde{h}_{a c}=-\left(1+\frac{2 m}{|\tilde{x}|}\right) \delta_{a c}+O_{k}\left(|\tilde{x}|^{-(1+\epsilon)}\right), \quad v=1-\frac{m}{|\tilde{x}|}+O_{k}\left(|\tilde{x}|^{-(1+\epsilon)}\right) \quad \text { as } \quad|\tilde{x}| \rightarrow \infty,
$$

where $|$.$| denotes the standard Euclidean norm. We refer to (\tilde{S}, \tilde{h}, v)$ as static vacuum data. The coefficient $m$ represents its ADM mass. In this article we shall only be interested in data with $\underset{\tilde{S}, \tilde{h})}{\operatorname{mass}} \neq 0$.

The pair $(\tilde{S}, \tilde{h})$ defines a particular type of time-reflection symmetric initial data for Einstein's vacuum field equations. Our interest in such initial data is motivated by observations which suggest that evolutions in time of time-reflection symmetric, asymptotically flat vacuum data admit at null infinity conformal extensions of prescribed smoothness if and only if the data behave in terms of a certain type of expansion at space-like infinity up to a certain order like static data (cf. 4] for a detailed discussion).

The full analysis of this relation requires detailed information on the asymptotic behaviour of static data and their conformal structures at space-like infinity. In previous work ([5]) we have given a complete characterization of static vacuum data in terms of a minimal set of symmetric trace free tensors at space-like infinity, referred to as null data. That article clarifies, in particular, the convergence problem. In the present article we take a first step towards characterizing conformal structures of static data. Obviously, such a characterization should be more easy if static data are related in a one to one fashion to their conformal structures. It will be shown that the relation is in fact more complicated.

A precise description of our result requires technical details. To keep the discussion short we refer the reader to [5] for further details. Beig and Simon ([2]) showed under certain assumptions, weakened later by Kennefick and O'Murchadha (7]), that static data with $m \neq 0$ admit conformal rescalings

$$
\tilde{h}_{a b} \rightarrow h_{a b}=\Omega^{2} \tilde{h}_{a b},
$$

with positive conformal factors $\Omega$ so that the set $S=\tilde{S} \cup\{i\}$, obtained by adjoining to $\tilde{S}$ a point $i$ representing space-like infinity, acquires a real analytic differentiable structure in which the conformal metric $h$ extends to a real analytic metric on $S$ and $\Omega$ extends to a function in $C^{2}(S) \cap C^{\omega}(\tilde{S})$ so that

$$
\Omega>0 \quad \text { on } \quad \tilde{S}, \quad \Omega=0, \quad d \Omega=0, \quad H_{e s s_{h}} \Omega=-2 h \quad \text { at } i .
$$

A particular example of such a conformal factor, determined by the static data themselves, is given by

$$
\Omega=\left(\frac{1-v}{m}\right)^{2}
$$

${ }^{1}$ The terms $O_{k}\left(|\tilde{x}|^{-(1+\epsilon)}\right)$ behave like $O\left(|\tilde{x}|^{-(1+\epsilon+j)}\right)$ under differentiations of order $j \leq k$. 
Let $(\tilde{S}, \tilde{h}, v)$ be a static vacuum data set with $m \neq 0$ and the fields $h, \Omega$ on $S$ related to it by (1.4), (1.6). We say that this set admits a non-trivial conformal rescaling if there exists another static vacuum data set $\left(\tilde{S}^{\prime}, \tilde{h}^{\prime}, v^{\prime}\right)$ with associated fields $h^{\prime}, \Omega^{\prime}$ on $S^{\prime}$ so that the following holds. Some neighbourhood $U^{\prime}$ of $i^{\prime}$ in $S^{\prime}$ can be identified diffeomorphically with some neighbourhood $U$ of $i$ in $S$, identifying $i^{\prime}$ with $i$, so that after identification there exists a non-constant smooth function $\vartheta>0$ on $U$ with

$$
h^{\prime}=\vartheta^{4} h .
$$

In other words, we require the existence of a conformal diffeomorphism which maps a neighbourhood of space-like infinity with respect to $\tilde{h}$ onto such a neighbourhood with respect to $\tilde{h}^{\prime}$ so that it extends in our gauge smoothly to a conformal map which maps $i$ onto $i^{\prime}$. The metrics $\tilde{h}, \tilde{h}^{\prime}$ are then related by

$$
\tilde{h}^{\prime}=\theta^{4} \tilde{h} \quad \text { with } \quad \theta=\left(\frac{1-v^{\prime}}{m^{\prime}}\right)^{-1} \vartheta \frac{1-v}{m} .
$$

In this article we investigate the question whether there exist static vacuum data sets which admit non-trivial conformal rescalings.

The following general transformations map static vacuum data sets onto such sets:

- Trival rescalings

$$
\tilde{h} \rightarrow \tilde{h}^{\prime}=\theta^{4} \tilde{h}, \quad v \rightarrow v^{\prime}=v \quad \text { with } \quad \theta=\text { const. }>0 .
$$

Asymptotic flatness of $\tilde{h}^{\prime}$ follows with the coordinate transformation $\tilde{x}^{a} \rightarrow \tilde{x}^{a^{\prime}}=\theta^{2} \tilde{x}^{a}$ in (1.3), which shows that the mass transforms as $m \rightarrow m^{\prime}=\theta^{2} m$. The corresponding conformal factor $\vartheta$ in (1.7) is given by $\vartheta=\frac{1-v}{m^{\prime}} \theta\left(\frac{1-v}{m}\right)^{-1}=\theta^{-1}$

- The transitions

$$
(\tilde{S}, \tilde{h}, v) \rightarrow\left(\tilde{S}, \tilde{h}^{\prime}=v^{4} \tilde{h}, v^{-1}\right) .
$$

under which the sign of the mass changes, $m \rightarrow m^{\prime}=-m$, and (1.7) holds with $\vartheta=1$. These transitions are suggested by the conformal static field equations studied below (cf. the remark following (2.8)). Without loss of generality it is therefore sufficient to consider the case

$$
m, m^{\prime}>0 .
$$

If the metric $\tilde{h}$ is conformally flat with non-vanishing mass it is necessarily induced by a Schwarzschild solution (4]). In isotropic coordinates $\tilde{x}^{a}$ the data are

$$
\tilde{h}=-\left(1+\frac{m}{2|\tilde{x}|}\right)^{4} \delta_{a b} d \tilde{x}^{a} d \tilde{x}^{b}, \quad v=\frac{1-\frac{m}{2|\tilde{x}|}}{1+\frac{m}{2|\tilde{x}|}},
$$

and (1.6) gives in the coordinates $x^{a}=|\tilde{x}|^{-2} \tilde{x}^{a}$, which are $h$-normal coordinates centered at $i$,

$$
h=-\delta_{a b} d x^{a} d x^{b}, \quad \Omega=\left(\frac{|x|}{1+\frac{m}{2}|x|}\right)^{2} .
$$


The transition $\tilde{h} \rightarrow \theta^{4} \tilde{h}, v \rightarrow v^{\prime}$ with $\theta=\frac{1+\frac{m^{\prime}}{2 \mid \tilde{x}}}{1+\frac{m}{2|\tilde{x}|}}, v^{\prime}=\frac{1-\frac{m^{\prime}}{2|\tilde{x}|}}{1+\frac{m^{\prime}}{2|\tilde{x}|}}$, which maps a Schwarzschild metric $\tilde{h}$ with mass $m$ onto such a metric with mass $m^{\prime}$, corresponds to a trivial rescaling combined with a coordinate transformation. In terms of $h$ this rescaling is given by (1.8) with $\vartheta=1$ and the information on the difference between the solutions is encoded in the conformal factors $\Omega$ and $\Omega^{\prime}$. The conformally flat case is special in admitting the 3-parameter group of special conformal transformations

$$
x^{a} \rightarrow \frac{x^{a}+d^{a} x_{c} x^{c}}{1+2 d_{c} x^{c}+d_{c} d^{c} x_{e} x^{e}}, \quad d^{a}=\text { const. } \in \mathbb{R}^{3},
$$

as local, non-trival, conformal maps of $h$ which leave $i$ fixed. In terms of the coordinates $\tilde{x}^{a}$ the map above is given by the simple translation $\tilde{x}^{a} \rightarrow \tilde{x}^{a}+d^{a}$. Conversely, the translations $x^{a} \rightarrow x^{a}+d^{a}$ is represented in terms of the coordinates $\tilde{x}^{a}$ by a special conformal transformation which maps a neighbourhood of infinity onto a punctured neighbourhood of the point $|d|^{-2} d^{a}$. In the following we shall be interested in static data which are not conformally flat.

The question posed above has been considered by Beig ([1]). He defines a certain quantity $Q$, quadratic in the Cotton tensor and its covariant derivatives up to second order, and shows that $h$ cannot admit non-trivial rescalings if $Q \neq 0$ at $i$. As also pointed out in [1, this condition excludes axi-symmetric static data. Unfortunately it is not clear what else is excluded. We wish to control the situation without imposing conditions and want to decide whether there do exist static, conformally non-flat vacuum data that admit non-trivial conformal rescalings. The lemmas proven in this article imply the following.

Theorem 1.1 Suppose $(\tilde{S}, \tilde{h}, v)$ are static vacuum data with ADM mass $m \neq 0$. If they admit a non-trival conformal rescaling, then $\tilde{h}$ admits a conformal Killing field $Y$ which is neither homothetic nor a Killing field for $\tilde{h}$ and, in addition, a Killing field $X$ which defines an infinitesimal axi-symmetry. The fields $Y$ and $X$ commute, are orthogonal to each other, and $Y$ is tangent to the axis defined by $X$ which passes through the point representing space-like infinity. Furthermore, if $\tilde{h}$ is not conformally flat it has a nonvanishing quadrupole moment.

There exists a 3-parameter family of static vacuum data with $m \neq 0$ which are not conformally flat and which do admit non-trival conformal rescalings. The associated spacetime metrics are of Petrov type $D$ along the axis and of Petrov type $I$ on an open neighbourhood surrounding the axis.

This result would provide complete information about the non-conformally flat static data with non-vanishing mass which admit non-trivial rescalings, were it not for an unanswered question. As discussed below, it is left open whether there exist data with nonvanishing quadrupole moment which admit non-trivial rescalings with $d \vartheta=0$ at $i$.

Apart from this omission the result above represents a 3-dimensional analogue of Brinkmann's theorem. Brinkmann studied the solutions of Einstein's vacuum field equations in 4 space-time dimensions which admit non-trivial conformal rescalings that yield new vacuum fields (asymptotic behaviour, however, played no role in these studies). He found them to be given by the solutions which have later been named vacuum pp-waves 
([3], [8]). These solutions are of Petrov type $N$. We note that the rescalings of the static data considered in this article do not extend to conformal rescalings of the correponding static vacuum space-times.

Details of the static data whose existence has been shown here will be discussed elsewhere.

\section{Conformal static vacuum field equations}

The derivation of the following equations has been discussed (in terms of $h$ and $\zeta=\rho / \mu$ ) in detail in [5. Using the conformal metric $h$ defined by (1.4), (1.6) and the function

$$
\rho=\left(\frac{1-v}{1+v}\right)^{2}
$$

one obtains the static vacuum equations (1.2) in the form

$$
\begin{gathered}
0=\Sigma_{a b}[h, \mu] \equiv D_{a} D_{b} \rho-s h_{a b}+\rho(1-\rho) s_{a b} \quad \text { with } \quad s=\frac{1}{3} \Delta_{h} \rho, \\
\left(\Delta_{h}-\frac{1}{8} R[h]\right)\left[\frac{1}{\sqrt{\rho}}\right]=\frac{4 \pi}{\sqrt{\mu}} \delta_{i} .
\end{gathered}
$$

Here $D$ denotes the covariant derivative defined by $h$, and the tensor

$$
s_{a b}=R_{a b}[h],
$$

is the Ricci tensor of $h$. It is trace free because the Ricci scalar of $h$ satisfies in the conformal gauge defined by (1.6)

$$
R[h]=0 .
$$

We note that the tensor $\frac{m}{2} s_{a b}(i)$ at space-like infinity represents the quadrupole moment of the static metric $\tilde{h}$. We set

$$
\mu=\frac{m^{2}}{4}
$$

and denote by $\delta_{i}$ the Dirac measure with weight one and support $\{i\}$, given by the standard Dirac measure $\delta_{0}$ in $h$-normal coordinates $x^{a}$ centered at $i$. In such coordinates the fields $h$ and $\rho$ are real analytic and satisfy

$$
\rho=0, \quad D_{a} \rho=0, \quad D_{a} D_{b} \rho=-2 \mu h_{a b} \quad \text { at } \quad i .
$$

The function $\rho$ can be characterized as the unique real analytic solution of (2.3), (2.6) or as the unique real analytic function satisfying (2.6) and

$$
2 \rho s=D_{a} \rho D^{a} \rho,
$$

which is a rewrite of (2.3). This equation can be shown (4]) to be in fact a consequence of (2.2) and (2.6) so that the essential information on the static field equations is encoded in (2.2), (2.6). 
In going from $h, \rho$ back to $\tilde{h}$ and $v$, one has to choose the sign of the square root. In the case of positive mass the correct formulas are

$$
\Omega=\frac{\rho}{\mu(1+\sqrt{\rho})^{2}}, \quad v=\frac{1-\sqrt{\rho}}{1+\sqrt{\rho}} .
$$

Replacing here the square root by its negative amounts to the transition (1.10).

The system (2.2) implies the integrability conditions

$$
0=\frac{1}{2} D^{e} \Sigma_{e a}=D_{a} s+(1-\rho) s_{a b} D^{b} \rho,
$$

and

$$
0=\frac{1}{\rho}\left(D_{[c} \Sigma_{a] b}+\frac{1}{2} D^{e} \Sigma_{e[c} h_{a] b}\right)=(1-\rho) D_{[c} s_{a] b}-2 D_{[c} \rho s_{a] b}-D^{d} \rho s_{d[c} h_{a] b},
$$

which extends by analyticity also to $i$.

While the static vacuum data are subject to a rescaling (1.7), the transformation of the potential was left unspecified. As pointed out above, the potential, represented by the function $\rho$, is determined in the asymptotically flat case uniquely by $\mu$ and $h$. The new potential should thus be given in terms of $\rho$, the conformal factor $\vartheta$, and the new mass term $\mu^{\prime}$.

Lemma 2.1 Assume that $(S, h, \rho)$ is derived from a static vacuum data set with mass $m>0$ as discussed above and $\vartheta>0$ is a $C^{2}$ function so that $h^{\prime}=\vartheta^{4} h$ is the conformal metric associated with static vacuum data $\tilde{h}^{\prime}, v^{\prime}$ on $S \backslash\{i\}$ with mass $m^{\prime}>0$. Set

$$
\nu=\frac{m^{2}}{m^{\prime 2} \vartheta(i)^{4}} .
$$

Then the function

$$
\rho^{\prime}=\frac{1}{\nu}\left(\frac{\vartheta}{\vartheta(i)}\right)^{2} \rho
$$

satisfies

$$
\left(\Delta_{h^{\prime}}-\frac{1}{8} R\left[h^{\prime}\right]\right)\left[\frac{1}{\sqrt{\rho^{\prime}}}\right]=\frac{4 \pi}{\sqrt{\mu^{\prime}}} \delta_{i},
$$

and relations (2.6) with $\rho, \mu, D$ replaced by $\rho^{\prime}, \mu^{\prime}$ and the covariant derivative operator $D^{\prime}$ defined by $h^{\prime}$. The function $\rho^{\prime}$ agrees with the one given by (2.1) with $v$ replaced by $v^{\prime}$.

Proof. Let $x^{a}$ resp. $x^{a^{\prime}}$ denote $h$ - resp. $h^{\prime}$-normal coordinates centered at $i$. A calculation then shows that the system $x^{a^{\prime}}$ satisfies, possibly after a rotation around the origin, the relation

$$
x^{a^{\prime}}=\delta^{a^{\prime}}{ }_{a}\left\{\vartheta(i)^{2} x^{a}-\vartheta(i)\left(\delta_{b c} \delta^{a d}-2 \delta^{a}{ }_{b} \delta^{d}{ }_{c}\right) \vartheta_{, d}(i) x^{a} x^{c}\right\}+O\left(\left|x^{a}\right|^{3}\right) .
$$


Writing the transformation $x^{a}=x^{a}\left(x^{c^{\prime}}\right)$ shortly $x=f\left(x^{\prime}\right)$, the transformation behaviour of Dirac distributions under coordinate transformations implies with the relation above $f^{*} \delta_{0}=\left|\operatorname{det} \frac{\partial f}{\partial x^{\prime}}(i)\right|^{-1} \delta_{0^{\prime}}=\vartheta(i)^{6} \delta_{0^{\prime}}([\underline{6}])$. With the conformal covariance of the conformal Laplacian and (2.3) it thus follows

$$
\begin{gathered}
f^{*}\left(\left(\Delta_{h^{\prime}}-\frac{1}{8} R_{h^{\prime}}\right)\left[\frac{1}{\sqrt{\rho^{\prime}}}\right]\right)=\sqrt{\frac{\mu}{\mu^{\prime}}} \vartheta(i)^{-1} f^{*}\left(\left(\Delta_{h^{\prime}}-\frac{1}{8} R_{h^{\prime}}\right)\left[\frac{1}{\vartheta \sqrt{\rho}}\right]\right) \\
=\sqrt{\frac{\mu}{\mu^{\prime}}} \vartheta(i)^{-1} f^{*}\left(\vartheta^{-5}\left(\Delta_{h}-\frac{1}{8} R_{h}\right)\left[\frac{1}{\sqrt{\rho}}\right]\right)=\frac{4 \pi}{\sqrt{\mu^{\prime}}} \delta_{0^{\prime}} .
\end{gathered}
$$

The relations (2.6) are verified by a direct calculation and the last statement follows by the uniqueness property pointed out above.

The quantity $\nu$ is left unchanged under trivial conformal rescalings. It has the following meaning. The conformal factor $\theta$ has in the coordinates of (1.3) in general an expansion

$$
\theta=\frac{1}{\vartheta(i)}\left(1+\frac{a}{|\tilde{x}|}+O\left(\frac{1}{|\tilde{x}|^{2}}\right)\right)
$$

with some coefficients $\vartheta(i)>0$ and $a$. Rescaling the metric (1.3) with $\theta$ one finds that the rescaled metric $\theta^{4} \tilde{h}$ acquires the mass $m^{\prime}=\vartheta(i)^{-2}(2 a+m)$. Using (2.8), (2.12) in the expression (1.8) for $\theta$, expanding $\rho$ using (1.5), and comparing with the expression for $\theta$ above, we find $2 a=m\left(\nu^{-1 / 2}-1\right)$ and thus again (2.11). A change of mass is thus generated purely by a trivial rescaling if $\nu=1$ but is partly due to an independent contribution if $\nu \neq 1$. We finally note the expressions

$$
\theta=\frac{\sqrt{\nu} \vartheta(i)+\sqrt{\rho} \vartheta}{(1+\sqrt{\rho}) \sqrt{\nu} \vartheta(i)^{2}}, \quad v^{\prime}=\frac{\sqrt{\nu} \vartheta(i)-\vartheta+(\sqrt{\nu} \vartheta(i)+\vartheta) v}{\sqrt{\nu} \vartheta(i)+\vartheta+(\sqrt{\nu} \vartheta(i)-\vartheta) v}
$$

for the conformal factor and the transformed potential. It follows that $\theta=1$ if and only if $\nu=1$ and $\vartheta=1$, while $v^{\prime}=v$ is equivalent to $(\sqrt{\nu} \vartheta(i)-\vartheta) v^{2}=(\sqrt{\nu} \vartheta(i)-\vartheta)$. This can hold only if $v=1$, which implies that $\tilde{h}_{a b}$ is flat, or if $\vartheta=\sqrt{\nu} \vartheta(i)$, which implies that $\nu=1, \vartheta=\vartheta(i)$, and $\theta=\vartheta(i)^{-1}$.

\section{The equations for the rescaling factor}

It will be convenient to replace $\vartheta$ by $\gamma \vartheta$ and assume

$$
\begin{gathered}
\vartheta(i)=1, \quad \gamma=\text { const. }>0, \quad \nu=\frac{\mu}{\mu^{\prime} \gamma^{4}}, \\
h^{\prime}=\gamma^{4} \vartheta^{4} h, \quad \rho^{\prime}=\frac{1}{\nu} \vartheta^{2} \rho .
\end{gathered}
$$

To derive conditions on the scaling factors we express

$$
\Sigma_{a b}\left[h^{\prime}, \mu^{\prime}\right] \equiv D_{a}^{\prime} D_{b}^{\prime} \rho^{\prime}-s^{\prime} h_{a b}^{\prime}+\rho^{\prime}\left(1-\rho^{\prime}\right) s_{a b}^{\prime},
$$


in terms of $h$ and $\rho$. With the general rescaling laws in 3 dimensions

$$
\begin{gathered}
R_{a b}\left[\vartheta^{4} h\right]=R_{a b}[h]-2 \vartheta^{-1} D_{a} D_{b} \vartheta+6 \vartheta^{-2} D_{a} \vartheta D_{b} \vartheta-2 h_{a b}\left\{\vartheta^{-1} D_{c} D^{c} \vartheta+\vartheta^{-2} D_{c} \vartheta D^{c} \vartheta\right\}, \\
\frac{1}{8} R\left[\vartheta^{4} h\right] \vartheta^{5}=-\Delta_{h} \vartheta+\frac{1}{8} R[h] \vartheta,
\end{gathered}
$$

where the right hand sides are expressed in terms of quantities derived from $h$, a direct calculation gives

$$
\begin{gathered}
\Sigma_{a b}\left[h^{\prime}, \mu^{\prime}\right]=\gamma^{4} \vartheta^{2} \frac{\mu^{\prime}}{\mu} \Sigma_{a b}[h, \mu]+\frac{8}{3} \gamma^{4} \frac{\mu^{\prime}}{\mu}\left(\frac{1}{\nu} \vartheta^{3} \rho^{2}-\vartheta \rho\right) \Delta_{h} \vartheta h_{a b} \\
-\gamma^{4} \frac{\mu^{\prime}}{\mu \nu^{2}} \vartheta^{6} \rho^{2}\left(D_{a} D_{b} u-\frac{1}{3} \Delta_{h} u h_{a b}+u(1-u) s_{a b}\right),
\end{gathered}
$$

where we set

$$
u=\nu \vartheta^{-2}
$$

and used the resulting relation

$$
2 u \Delta_{h} u-3 D_{c} u D^{c} u=-4 \nu^{2} \vartheta^{-5} \Delta_{h} \vartheta .
$$

Equation (3.4) implies with $R[h]=0$ and $R\left[h^{\prime}\right]=0$

$$
\Delta_{h} \vartheta=0
$$

From these relations we read off the following basic condition.

Lemma 3.1 Suppose $h$ and $\rho$ satisfy (2.2) and (2.6) with some constant $\mu>0$. If $\mu^{\prime}, \gamma>0$ are constants and $\vartheta$ a positive function with $\vartheta(i)=1$, then $h^{\prime}=\gamma^{4} \vartheta^{4} h$ and $\rho^{\prime}=\frac{1}{\nu} \vartheta^{2} \rho$ with $\nu=\frac{\mu}{\mu^{\prime} \gamma^{4}}$ satisfy $\Sigma_{a b}\left[h^{\prime}, \mu^{\prime}\right]=0$ if and only if $u=\nu \vartheta^{-2}$ satisfies the overdetermined system

$$
\begin{gathered}
0=\Pi[h, u] \equiv 2 u t-D_{c} u D^{c} u \quad \text { with } \quad t=\frac{1}{3} \Delta_{h} u, \\
0=\Pi_{a b}[h, u] \equiv D_{a} D_{b} u-t h_{a b}+u(1-u) s_{a b} .
\end{gathered}
$$

Moreover, $u$ must satisfy the initial condition

$$
u(i)=\nu .
$$

Using the Bianchi identity and the decomposition $R_{d b c a}=2\left(h_{d[c} s_{a] b}-h_{b[c} s_{a] d}\right)$, which holds because $R[h]=0$ and $\operatorname{dim}(S)=3$, one gets from (3.9) the integrability conditions

$$
\begin{gathered}
0=\frac{1}{2} D^{c} \Pi_{c a}=D_{a} t+(1-u) D^{c} u s_{c a} \\
0=\frac{1}{u}\left(D_{[c} \Pi_{a] b}+\frac{1}{2} D^{d} \Pi_{d[c} h_{a] b}\right)=(1-u) D_{[c} s_{a] b}-2 D_{[c} u s_{a] b}-D^{d} u s_{d[c} h_{a] b} .
\end{gathered}
$$


The identity

$$
D_{a} \Pi=u D^{c} \Pi_{c a}-2 D^{c} u \Pi_{c a},
$$

implies that (3.8) will be satisfied by a solution $u$ of (3.9) if $\Pi(i)=0$ i.e. if

$$
u(i)=\nu>0, \quad 2 \nu t(i)=c_{a} c^{a} \quad \text { with } \quad c_{a}=D_{a} u(i) .
$$

Let $x(\tau)$ denote a geodesic through $i$ with unit tangent vector $\dot{x}(\tau)$. Transvecting equations (3.9), (3.11) suitably with $\dot{x}$, one obtains a system of ODE's for $u, D_{a} u, t$ along this curve, which shows that a solution $u$ of (3.9), (3.11), if it exists, must be analytic and uniquely determined by the data

$$
u(i)=\nu>0, \quad \dot{u}(i)=\dot{x}^{a} c_{a}, \quad t(i)=\frac{1}{2 \nu} c_{a} c^{a} .
$$

The function $u$ so obtained will in general not satisfy the complete system (3.9). It will be shown that the existence of non-trivial solutions to equation (3.9) imposes strong restrictions on the metric $h$. Because of the factor $1-u$ in equations (3.9), (3.11) it follows immediately that

$$
u \equiv 1 \quad \text { if } \quad \nu=1, \quad c_{a}=0 .
$$

The following result will be useful later.

Lemma 3.2 Let $u$ be a solution to (3.9), (3.14) on a neighbourhood of $i$ on which $\rho<a$ for some $a>0$. If $u=F(\rho)$ with some function $F \in C^{2}([0, a[)$, then $u$ is the trivial solution $u=\nu$. Moreover, $h$ is flat unless $\nu=1$.

Proof. Observing (2.7) one gets

$$
2 u t-D_{c} u D^{c} u=\frac{1}{3} D_{a} \rho D^{a} \rho\left(\frac{3}{\rho} F F^{\prime}+2 F F^{\prime \prime}-3\left(F^{\prime}\right)^{2}\right),
$$

so that (3.8) is equivalent to the ODE

$$
\frac{3}{\rho} F F^{\prime}+2 F F^{\prime \prime}-3\left(F^{\prime}\right)^{2}=0 .
$$

If $F^{\prime}=0$ at a point where $F>0$ it follows that $u=F=$ const. $=\nu$ which implies with (3.9) that $s_{a b}=0$ unless $\nu=1$. Near a point where $F>0$ and $F^{\prime} \neq 0$ the equation above implies $0=\frac{3}{\rho}+2 \frac{F^{\prime \prime}}{F^{\prime}}-3 \frac{F^{\prime}}{F}=\left(\log \frac{\rho^{3}\left(F^{\prime}\right)^{2}}{F^{3}}\right)^{\prime}$ whence $F=\frac{\rho}{(b+d \sqrt{\rho})^{2}}$ with $b, d=$ const. $>0$, $b \neq 0$. But then $u(p)=F(\rho(p)) \rightarrow 0 \neq \nu$ as $p \rightarrow i$, which contradicts our assumptions.

\section{Implications for $h$}

Let $\tilde{h}, v$ be static data and $h, \rho$ the associated conformal fields. We shall discuss now properties of $\tilde{h}$ and $h$ which are implied by the existence of a non-trivial solution $u$ to (3.8), (3.9), (3.10). Assume that

$U$ is an $i$-centered, convex $h$-normal nbhd so that $0<\rho<1, D_{a} \rho \neq 0$ on $U \backslash\{i\}$, (4.1) 
and set with a given function $u$

$$
\rho_{a}=D_{a} \rho, \quad u_{a}=D_{a} u, \quad w=\frac{1-u}{1-\rho}, \quad w_{a}=D_{a} w, \quad U^{*}=\left\{p \in U \mid w_{a}(p) \neq 0\right\},
$$

so that $i \in U^{*}$ if and only if $u_{a}(i) \neq 0$. We recall that $h$ is conformally non-flat if and only if the set $\left\{p \in U \mid s_{a b}(p) \neq 0\right\}$ is dense in $U([4])$.

Lemma 4.1 Let $h, \rho$ denote a solution to (2.2), (2.6) which is not conformally flat. Suppose $u$ is a non-constant, positive solution to (3.8), (3.9), (3.10) on a set $U$ satisfying (4.1) and define $w$ as in (4.2). Then the set $U^{*}$ is dense in $U$ and there exists a smooth function $\beta$ on $U^{*}$ so that

$$
s_{a b}=\beta\left(w_{a} w_{b}-\frac{1}{3} h_{a b} w_{c} w^{c}\right) .
$$

If $V \subset U^{*}$ is a connected, simply connected neighbourhood of a point $p \in U^{*}$, there exist a constant $\beta_{*} \neq 0$ and a function $H=H(w)$ defined on $V$ with $H(w(p))=0$ so that the Ricci tensor has on $V$ the representation

$$
s_{a b}=\frac{\beta_{*}}{1-\rho} e^{H}\left(w_{a} w_{b}-\frac{1}{3} h_{a b} w_{c} w^{c}\right) .
$$

If $u_{a}(i) \neq 0$ we can choose $p=i$.

Proof. If $w_{a}$ vanished on an open subset of $U, w$ would be constant on $U$ because $u$ and $\rho$ are analytic. It would follow that $u=\nu+\rho(1-\nu)$ whence $u=$ const. by lemma 3.2, in conflict with our assumptions. It follows that $U^{*}$ is dense in $U$. Because

$$
\begin{gathered}
\frac{1}{u(1-\rho)}\left(D_{[c} \Pi_{a] b}+\frac{1}{2} D^{d} \Pi_{d[c} h_{a] b}\right)= \\
\frac{1}{u(1-\rho)}\left(D_{[c} \Pi_{a] b}+\frac{1}{2} D^{d} \Pi_{d[c} h_{a] b}\right)-\frac{1-u}{\rho(1-\rho)^{2}}\left(D_{[c} \Sigma_{a] b}^{*}+\frac{1}{2} D^{e} \Sigma_{e[c}^{*} h_{a] b}\right) \\
=2 w_{[c} s_{a] b}+w^{d} s_{d[c} h_{a] b},
\end{gathered}
$$

equation (3.12) holds on $U^{*}$ if and only if

$$
2 w_{[c} s_{a] b}+w^{d} s_{d[c} h_{a] b}=0 .
$$

Contraction with $2 w^{c}$ gives

$$
2 w_{c} w^{c} s_{a b}-2 w_{a} s_{c b} w^{c}+w^{d} s_{d c} w^{c} h_{a b}-w^{d} s_{d a} w_{b}=0 .
$$

The antisymmetric part of this equation reads $w^{d} s_{d[a} w_{b]}=0$, which implies on $U^{*}$

$$
w^{d} s_{d a}=\alpha w_{a},
$$

with some function $\alpha$. Using this in the equation above, we obtain (4.3), which satisfies (4.5) without restriction on $\beta$. 
With (2.2) and (3.9) one obtains

$$
D_{a} w_{b}=J h_{a b}+(1-u)(1-w) s_{a b}+\frac{1}{1-\rho}\left(w_{a} \rho_{b}+w_{b} \rho_{a}\right) \quad \text { with } \quad J=\frac{w s-t}{1-\rho} .
$$

It follows with (4.3)

$$
\begin{gathered}
D_{a} w^{a}=3 J+\frac{2}{1-\rho} \rho_{a} w^{a}, \\
w^{a} D_{b} w_{a}=J w_{b}+\frac{2}{3}(1-u)(1-w) \beta w_{c} w^{c} w_{b}+\frac{1}{1-\rho}\left(w_{a} w^{a} \rho_{b}+w_{b} \rho_{a} w^{a}\right), \\
w^{b} w^{a} D_{b} w_{a}=\left\{J+\frac{2}{3}(1-u)(1-w) \beta w_{c} w^{c}+\frac{2}{1-\rho} \rho_{a} w^{a}\right\} w_{c} w^{c} .
\end{gathered}
$$

On $U^{*}$ the Bianchi identity and the gauge condition $R[h]=0$ imply with (4.3)

$$
0=D^{a} s_{a b}=w^{a} D_{a} \beta w_{b}-\frac{1}{3} D_{b} \beta w_{c} w^{c}+\beta\left(D_{a} w^{a} w_{b}+\frac{1}{3} w^{c} D_{b} w_{c}\right) .
$$

After contraction with $w^{b}$ this can be solved for $w^{a} D_{a} \beta$. Inserting the resulting expression again into the equation, gives

$$
\begin{gathered}
w_{c} w^{c} D_{a} \beta=\beta\left\{-\frac{3}{2}\left(D_{c} w^{c}+\frac{w^{b} w^{c} D_{b} w_{c}}{w_{c} w^{c}}\right) w_{a}+w^{c} D_{a} w_{c}\right\} \\
=\beta\left\{-5 J w_{a}-\frac{5}{1-\rho} \rho_{c} w^{c} w_{a}-\frac{1}{3}(1-u)(1-w) \beta w_{c} w^{c} w_{a}+\frac{1}{1-\rho} w_{c} w^{c} \rho_{a}\right\} .
\end{gathered}
$$

and thus finally

$$
D_{a} \beta=\beta\left\{\frac{1}{1-\rho} \rho_{a}+K w_{a}\right\}
$$

with

$$
w_{c} w^{c} K=-5 J-\frac{5}{1-\rho} \rho_{c} w^{c}-\frac{1}{3}(1-u)(1-w) w_{c} w^{c} \beta .
$$

The relation $\beta(p)=0$ would imply with (4.9) that $\beta=0$, whence $s_{a b}=0$ on a neighbourhood of $p$ in $U^{*}$ and thus $s_{a b}=0$ on $U$ by analyticity, contradicting conformal non-flatness. Thus $\beta(p) \neq 0$, whence $\beta \neq 0$ on $V$, and equation (4.9) can be written there in the form $D_{a} \log |(1-\rho) \beta|=K D_{a} w$. This implies that $D_{[a} K D_{b]} w=0, K$ can be written as a function of $w$ on $V$, and there exists a function $H=H(w)$ with $H(w(p))=0$ so that $D_{a}(\log |(1-\rho) \beta|-H)=0$, whence, with $\beta_{*}=\left.(1-\rho) \beta\right|_{p} \neq 0$,

$$
\beta=\frac{\beta_{*}}{1-\rho} e^{H} \quad \text { on } \quad V .
$$

To state the following result, we note that lemma 3.2 implies under the assumptions of lemma 4.1 that $\epsilon^{a b c} u_{b} \rho_{c} \neq 0$ on a dense open subset of $U$. 
Lemma 4.2 Assume the notation and the assumptions of lemma 4.1. If $V$ is chosen so that $\epsilon^{a b c} u_{b} \rho_{c} \neq 0$ on $V$, there exists a function $l=l(w)$ on $V$ so that

$$
X^{a}=l \epsilon^{a b c} u_{b} \rho_{c},
$$

defines a non-trivial Killing field for $h$ on $V$. It extends to an analytic, hypersurface orthogonal Killing field $X$ on $U$ which satisfies the relations

$$
X^{a} \rho_{a}=0, \quad X^{a} u_{a}=0,
$$

and is a Killing field for $\tilde{h}$ on $U \backslash\{i\}$. Independent of the choice of $V$ it is determined uniquely up to a constant real factor. It vanishes at $i$ and defines an infinitesimal axisymmetry with closed integral curves near $i$. If $u_{a}(i) \neq 0$ its axis is given by the $h$-geodesic $\gamma(\tau)$ with tangent vector $u^{a}(i)$ at $i$.

Proof. With the expression (4.11) a direct calculation using (2.2), (3.9), (4.3), (4.10) gives on $V$

$$
D_{a} X_{b}=\epsilon_{a b}^{c} \eta_{c}+l^{-1}\left\{D_{b} l+l \beta_{*} e^{H}\left(w-w^{2}\right) w_{b}\right\} X_{a},
$$

where

$$
\eta_{c}=\frac{1}{3} l\left\{\left(\Delta_{h} \rho+\rho(1-\rho) \beta w_{d} w^{d}\right) u_{c}-\left(\Delta_{h} u+u(1-u) \beta w_{d} w^{d}\right) \rho_{c}\right\} .
$$

With the choice

$$
l=l_{*} e^{L(w)} \quad \text { where } L \text { satisfies } \quad L^{\prime}(w)=-\beta_{*} e^{H}\left(w-w^{2}\right) \quad \text { and } \quad l_{*}=\text { const. }>0,
$$

this implies

$$
D_{a} X_{b}=\epsilon_{a b}^{c} \eta_{c}
$$

It follows

$$
D_{(a} X_{b)}=0, \quad \epsilon^{a b c} X_{a} D_{b} X_{c}=0 .
$$

Equations (4.12) are an immediate consequence of the definition of $X$. Since then $X^{a} D_{a} \Omega=$ 0 by (2.8), $X$ is also a Killing field for the metric $\tilde{h}$.

To construct the extension of $X$ to $U$ we use the integrability condition for the Killing equation,

$$
D_{a} D_{b} X_{c}=X_{d} R_{a b c}^{d}
$$

which is satisfied by $X$ on $V$ and to be satisfied on $U$. Fix $p \in V$. The geodesics $\gamma(\tau)$ with $\gamma(0)=p$ cover the convex normal neighbourhood $U$. The ODE's

$$
D_{\dot{\gamma}}^{2} X_{c}=X_{d} R_{a b c}^{d} \dot{\gamma}^{a} \dot{\gamma}^{b}
$$

along these geodesics determine a unique analytic extension of $X$ to $U$. The Killing and the hypersurface orthogonality conditions (4.16) as well as the relations (4.12) extend to $U$ by analyticity. As above it follows that the extended field is also a Killing field for $\tilde{h}$.

The relation $X^{[a} \epsilon^{b] c d} u_{c} \rho_{d}=0$, which holds on $V$, extends to $U$ by analyticity so that

$$
X^{a} \sim \epsilon^{a c d} u_{c} \rho_{d} \quad \text { on } \quad U .
$$


The factor $l$ which relates these fields on $V$ needs a priori not be bounded on $U$ but up to a constant factor the Killing field $X^{a}$ is determined uniquely.

The relation $\mathcal{L}_{X} \rho=0$ on $U$ implies

$$
0=D_{a} \mathcal{L}_{X} \rho=\mathcal{L}_{X} D_{a} \rho=X^{b} D_{b} D_{a} \rho+D_{a} X^{b} D_{b} \rho,
$$

which reduces at $i$ to

$$
0=-2 \mu X_{a}(i)
$$

It follows that $D_{a} X_{b} \neq 0$ at $i$, otherwise $X$ would vanish identically. Any geodesic through $i$ is mapped by the flow of $X$ onto such geodesic because $i$ is a fixed point of the flow. Because $\operatorname{dim}(S)=3$ and $D_{a} X_{b}$ is anti-symmetric there exists a tangent vector $t^{a} \neq 0$ at $i$ with $t^{a} D_{a} X_{b}=0$. This vector is invariant under the flow of $X$. The geodesic $\gamma(\tau)$ satisfying $\gamma(0)=i$ and $\dot{\gamma}(0)=t$ is thus pointwise invariant under the flow so that $\left.X\right|_{\gamma(\tau)}=0$ and the points of $\gamma(\tau)$ represent the axis of $X$. Because the flow of $X$ preserves orthogonality and maps geodesics onto such, it maps any geodesic orthogonal to $\gamma$ onto another such geodesic. Since it preserves affine parameters it follows that the flow lines of $X$ are closed near $i$.

If $u_{a}(i) \neq 0$ the function $\beta$ given by (4.10) and thus the function $l$ can be given on a neighbourhood of $i$ so that $l=l_{*}$ and $e^{H}=1$ at $i$ and the expressions (4.11) and (4.15) with (4.13) can be assumed to hold on this neighbourhood. While then $X^{a}=0$ at $i$, we have $\eta_{c}(i)=-2 l_{*} u_{c}$, so that $\left.D_{a} X_{b}\right|_{i}=-2 l_{*} \epsilon_{a b}{ }^{c} u_{c}(i) \neq 0$ and $\left.u^{a} D_{a} X_{b}\right|_{i}=0$. It follows that the axis is given by the geodesic with tangent vector $u^{a}$ at $i$.

If $u_{a}(i) \neq 0$ we normalize $X$ by setting

$$
l_{*}=\frac{1}{2 c} \quad \text { with } \quad c=\left.\sqrt{-u_{c} u^{c}}\right|_{i}>0 \quad \text { so that }\left.\quad \eta_{a} \eta^{a}\right|_{i}=-1 .
$$

It follows with (4.17) that $\eta_{a} \eta^{a}=D_{a} X_{b} D^{a} X^{b} / 2=$ const. $=-1$ along the the axis, where $X$ vanishes. The flow of $X$ induces then rotations of the tangent space $T_{i} S$ with period $2 \pi$ and not smaller. If $\phi$ denotes the natural parameter on the integral curves of $X$ which vanishes on a hypersurface orthogonal to $X$ which approaches the axis from one side, it defines a coordinate with hypersurfaces $\{\phi=$ const. $\} \perp X$ and the integral curves of $X$ close exactly if $\phi \in[0,2 \pi[$.

Lemma 4.3 Assume the notation and the assumptions of lemmas 4.1, 4.2. The field

$$
Y^{a}=f w^{a} \quad \text { with } \quad f=\frac{l}{l_{*}}(1-\rho)^{2},
$$

satisfies on $V$

$$
D_{a} Y_{b}=\omega h_{a b}+\frac{1}{l_{*}} \epsilon_{a b}{ }^{c} X_{c} \quad \text { with } \quad \omega=f\left(J-\frac{1}{3}\left(w-w^{2}\right) w_{c} w^{c} \beta_{*} e^{H}\right) .
$$

It extends to an analytic, hypersurface orthogonal field $Y$ on $U$ satisfying the conformal Killing equation

$$
D_{a} Y_{b}+D_{b} Y_{a}=2 \omega h_{a b}
$$


and the relations

$$
X_{a} Y^{a}=0, \quad[X, Y]=0 .
$$

It is tangential to the axis through $i$ defined by $X$, where it does not vanish. It is a conformal Killing field but neither homothetic nor a Killing field for $\tilde{h}$. Independent of the choice of $V$ the extended field is unique up to a non-vanishing constant real factor.

Proof. With (4.7) we get on $V$

$$
\begin{gathered}
D_{a} Y_{b}=e^{L}\left((1-\rho)^{2} L^{\prime} w_{a}-2(1-\rho) \rho_{a}\right) w_{b} \\
+f\left(J h_{a b}+(1-u)(1-w) \beta\left(w_{a} w_{b}-\frac{1}{3} h_{a b} w_{c} w^{c}\right)+\frac{1}{1-\rho}\left(w_{a} \rho_{b}+w_{b} \rho_{a}\right)\right) \\
=f\left(J-\frac{1}{3}\left(w-w^{2}\right) w_{c} w^{c} \beta_{*} e^{H}\right) h_{a b}+(1-\rho) e^{L}\left(w_{a} \rho_{b}-w_{b} \rho_{a}\right), \\
=\omega h_{a b}-e^{L}\left(u_{a} \rho_{b}-u_{b} \rho_{a}\right)=\omega h_{a b}+\frac{1}{l_{*}} \epsilon_{a b}{ }^{c} X_{c},
\end{gathered}
$$

which implies (4.23), (4.24) on $V$. Hypersurface orthogonality and orthogonality to $X$ follow immediately from the definitions of $X$ and $Y$. The second of relations (4.25) follows by a direct calculation from

$$
\begin{gathered}
{[X, Y]_{a}=X^{c} D_{c} Y_{a}-Y^{c} D_{c} X_{a}=\omega X_{a}-f w^{c} \epsilon_{c a d} \eta^{d}} \\
=\omega X_{a}-f \frac{1}{3}\left[\left(\Delta_{h} \rho+\rho w_{d} w^{d} \beta_{*} e^{H}\right) \frac{1-u}{(1-\rho)^{2}}-\left(\Delta_{h} u+u w w_{d} w^{d} \beta_{*} e^{H}\right) \frac{1}{1-\rho}\right] X_{a} \\
=\omega X_{a}-f\left[J-\frac{1}{3} \frac{u-\rho}{1-\rho} w w_{d} w^{d} \beta_{*} e^{H}\right] X_{a}=0 .
\end{gathered}
$$

To extend $Y$ to $U$ we consider the integrability conditions for the conformal Killing equations $([9])$,

$$
D_{a} D_{b} Y_{c}=Y_{d} R_{a b c}^{d}+\omega_{a} h_{b c}+\omega_{b} h_{a c}-\omega_{c} h_{a b},
$$

with

$$
D_{a} \omega=\omega_{a},
$$

and

$$
D_{a} \omega_{b}=-\mathcal{L}_{Y} s_{a b}=-\left(Y^{c} D_{c} s_{a b}+D_{a} Y^{c} s_{c b}+D_{b} Y^{c} s_{a c}\right),
$$

satisfied in our conformal gauge by $Y$ on $V$ and to be satisfied on $U$. Fix $p \in V$ and consider the geodesics through $p$. The equations above imply a linear system of ODE's for $Y_{a}, D_{a} Y_{b}, \omega, \omega_{a}$ along the geodesics which determine a unique analytic extension of $Y$ to $U$. Equations (4.24) and (4.25) extend to $U$ by analyticity.

Because

$$
X^{a}=-\frac{l_{*}}{1-\rho} \epsilon^{a b c} Y_{b} \rho_{c},
$$

the relation $D_{a} X_{b}(i) \neq 0$ implies that $Y^{a} \neq 0$ at $i$ and thus also on the axis near $i$. The restriction of

$$
0=[X, Y]_{a}=X^{c} D_{c} Y_{a}-Y^{c} D_{c} X_{a},
$$


to the axis implies that $Y$ is tangent to the axis, the conformal Killing equation implies that $Y \neq 0$ there.

It holds $\mathcal{L}_{Y} \tilde{h}=\mathcal{L}_{Y}\left(\Omega^{-2} h\right)=2 \tilde{\omega} \tilde{h}$ with $\tilde{\omega}=\omega-\Omega^{-1} Y^{a} D_{a} \Omega$. Because $\left.D_{a}\left(Y^{c} \rho_{c}\right)\right|_{i}=$ $-2 \mu Y_{a}(i) \neq 0$, it follows with (2.8) that $\Omega^{-1} Y^{a} D_{a} \Omega$ diverges at $i$. Thus $\tilde{\omega}$ can neither be constant nor vanish.

On $V$, which can be chosen to contain $i$ if $u_{a}(i) \neq 0$, the dualized version of the Cotton tensor $B_{b c a}=D_{[c} R_{a] b}$ acquires by (2.10), (4.4), (4.11), and (4.22) the concise form

$$
B_{a b}=\frac{1}{2} B_{a c d} \epsilon_{b}{ }^{c d}=\frac{1}{1-\rho} s_{d(a} \epsilon_{b)}{ }^{c d} \rho_{c}=\frac{\beta_{*} e^{H-2 L}}{l_{*}(1-\rho)^{5}} X_{(a} Y_{b)} .
$$

The conformal factor (2.8) and the transformation laws under conformal rescalings give

$$
R_{a b}[\tilde{h}]=\sqrt{\rho} \beta\left(w_{a} w_{b}-\frac{1}{3} h_{a b} w_{c} w^{c}\right)-\frac{3}{2 \sqrt{\rho}^{3}(1+\sqrt{\rho})^{2}}\left(\rho_{a} \rho_{b}-\frac{1}{3} \rho_{c} \rho^{c} h_{a b}\right) .
$$

At $i$ holds $w_{a} \rho^{a}=0$ and $D_{c}\left(w_{a} \rho^{a}\right)=2 \mu u_{c}(i) \neq 0$ if $u_{c}(i) \neq 0$. Thus, if $u_{c}(i) \neq 0$, there exists a smooth hypersurface $H$ through $i$ on which $w_{a} \rho^{a}=0$. With $X_{a} \rho^{a}=0$, $X_{a} w^{a}=0$ it follows that the vector fields $w^{a}, \rho^{a}, X^{a}$ define on $H \backslash\{i\}$ an orthogonal set of eigenvectors of $R_{a b}[\tilde{h}]$. The direct calculation shows that the three eigenvalues are different from each other. It follows that $\tilde{g}$ is of Petrov type $I$ near $H \backslash\{i\}$. The fields $\rho^{a}$ and $w^{a}$ are proportional to $u^{a}$ on the axis because $X=0$ there. It follows that $R_{a b}[\tilde{h}] \sim \rho_{a} \rho_{b}-\frac{1}{3} \rho_{c} \rho^{c} h_{a b}$ so that $\tilde{g}$ is of Petrov type $D$ along the axis (cf. [8]).

\section{Existence and non-existence results}

In spite of the simple conclusion (3.15) the case were $u_{a}(i)=0$ is not easily discussed in general. The function $\beta=\frac{3}{2} \frac{s_{a b} w^{a} w^{a}}{\left(w_{c} w^{c}\right)^{2}}$ in (4.3) may become, along with the quantities $H$ and $L$, singular at $i$ if $w_{a}(i)=0$. In fact, if $s_{a b}$ does not vanish at $i$, it is there due to axi-symmetry of the form

$$
s_{a b}(i)=\xi\left(n_{a} n_{b}+\frac{1}{3} h_{a b}\right), \quad \xi \neq 0,
$$

with a unit vector $n^{a}$ pointing in the direction of the axis. With this one finds that $\beta=-\operatorname{sign}(\xi) \sqrt{3 / 2 s_{e d} s^{e d}}\left(w_{c} w^{c}\right)^{-1}$ is unbounded near $i$. If $D_{d}$ is applied to equations (2.10) and (3.12), the resulting equations are subtracted from each other, and the difference is restricted to $i$, one obtains with $u_{a}(i)=0$ and (2.2), (2.6), (3.8), (3.9) at $i$ the relation

$$
\nu\left\{2 s_{d[c} s_{a] b}+s_{d}{ }^{f} s_{f[c} h_{a] b}\right\}=2 \mu\left\{2 h_{d[c} s_{a] b}+s_{d[c} h_{a] b}\right\} .
$$

It restricts the parameter in (5.1) by $\xi=\frac{6 \mu}{\nu}$ but it does not exclude (5.1). We shall leave the case $D_{a} u(i)=0, s_{a b}(i) \neq 0$ open in this article. If the second of these conditions is dropped we get with (3.15) a complete, though negative answer. 
Lemma 5.1 Suppose $h, \rho$ is a solution to the conformal static vacuum equations (2.2), (2.6) such that $s_{a b}(i)=0$. Then a positive solution $u$ to equations (3.8), (3.9), (3.10) near $i$ which satisfies $u(i)=\nu \neq 1$ and $D_{a} u(i)=0$ is the constant solution $u=\nu$ and $h$ is flat.

Proof. We assume that $u$ is not a constant and show that this leads to a contradiction. In the following results of [5] will be used. For the notions set below in quotation marks and statements relating to them we refer the reader to that article.

Let $x^{a}$ denote $h$-normal coordinates centered at $i$, so that $h_{a b}^{*} \equiv h_{a b}(i)=-\delta_{a b}$. Equation (2.10) implies with (2.6) that $D_{a} s_{b c}(i)$ is totally symmetric. More generally, if

$$
s_{a b}=O\left(|x|^{k}\right) \text { for some } k \geq 1,
$$

the 'exact set of equations argument' implies with (2.10) that the tensor

$$
\psi_{a_{1} \ldots a_{k} a b} \equiv D_{a_{1}} \ldots D_{a_{k}} s_{a b}(i),
$$

is given in space spinor notation by a completely symmetric spinor $\psi_{A_{1} B_{1} \ldots A_{k+2} B_{k+2}}$ because it either vanishes or defines the non-vanishing 'null datum' of lowest order for the solution $h$. We shall show that it vanishes so that in fact $s_{a b}=O\left(|x|^{k+1}\right)$.

Equations (3.8), (3.9), (3.11) imply with the assumption above

$$
u=\nu+O\left(|x|^{k+2}\right), \quad D_{a_{1}} \ldots D_{a_{k+2}} u(i)=-\nu(1-\nu) \psi_{a_{1} \ldots a_{k+2}},
$$

so that one obtains at $i$ 'normal expansions'

$$
\begin{gathered}
s_{a b}=\frac{1}{k !} \psi_{a_{1} \ldots a_{k} a b} x^{a_{1}} \ldots x^{a_{k}}+O\left(|x|^{k+1}\right), \\
u=\nu-\nu(1-\nu) \frac{1}{(k+2) !} \psi_{a_{1} \ldots a_{k+2}} x^{a_{1}} \ldots x^{a_{k+2}}+O\left(|x|^{k+3}\right), \\
D_{a} u=-\nu(1-\nu) \frac{1}{(k+1) !} \psi_{a_{1} \ldots a_{k+1} a} x^{a_{1}} \ldots x^{a_{k+1}}+O\left(|x|^{k+2}\right), \\
\rho=-\mu h_{a b}^{*} x^{a} x^{b}+O\left(|x|^{k+4}\right), \quad D_{a} \rho=-2 \mu h_{a b}^{*} x^{b}+O\left(|x|^{k+3}\right),
\end{gathered}
$$

whence

$$
w_{a}=\frac{-2 \mu(1-\nu) h_{a b}^{*} x^{b}}{\left(1+\mu h_{a b}^{*} x^{a} x^{b}\right)^{2}}+O\left(|x|^{k+1}\right), \quad w_{a} w^{a}=\frac{(2 \mu(1-\nu))^{2} h_{a b}^{*} x^{a} x^{b}}{\left(1+\mu h_{a b}^{*} x^{a} x^{b}\right)^{4}}+O\left(|x|^{k+2}\right) .
$$

It follows that $w_{a} \neq 0$ on a punctured neighbourhood of $i$. Consider near $i$ the real analytic field

$$
f_{a b}=2\left(w_{c} w^{c}\right)^{2} s_{a b}-3 w^{c} w^{d} s_{c d}\left(w_{a} w_{b}-\frac{1}{3} h_{a b} w_{e} w^{e}\right) .
$$

Lemma4.1 implies with the assumption that $u$ is not constant that the field $f_{a b}$ vanishes on the open set where $w_{a} \neq 0$ and thus, by analyticity, everywhere. In the normal coordinates $x^{a}$ we extend near $i$ now all real analytic fields holomorphically into the complex domain and consider a complex null geodesic $x^{a}(\tau)=\tau l^{a}$ with $l^{a}=$ const. $\neq 0, l_{a} l^{a}=0$. 
Expanding $f_{a b}(x(\tau))$ at $\tau=0$ and observing the expansions above gives

$$
0=f_{a b}=-3 \frac{(2 \mu(1-\nu))^{4}}{k !} \psi_{a_{1} \ldots a_{k+2}} l^{a_{1}} \ldots l^{a_{k+2}} l_{a} l_{b} \tau^{k+4}+O\left(|\tau|^{k+5}\right) .
$$

This shows that

$$
0=\psi_{a_{1} \ldots a_{k+2}} l^{a_{1}} \ldots l^{a_{k+2}}=\psi_{A_{1} B_{1} \ldots A_{k+2} B_{k+2}} \iota^{A_{1}} \ldots \iota^{B_{k+2}},
$$

where $l^{a}$ is represented on the right hand side by the spinor $\iota^{A} \iota^{B}$. Because $l^{a}$ and thus the spinor $\iota^{A}$ is arbitrary here, the symmetric spinor $\psi_{A_{1} B_{1} \ldots A_{k+2} B_{k+2}}$ must vanish, which implies $s_{a b}=O\left(|x|^{k+1}\right)$ and $u=\nu+O\left(|x|^{k+3}\right)$. In contradiction to our assumption it follows inductively that $u-\nu$ and $s_{a b}$ vanish at $i$ at all orders.

The existence result anounced in the introduction will now be proven. In stating it we ignore trivial rescalings.

Lemma 5.2 For given data $\mu, \nu, \beta_{*} \in \mathbb{R}$ and $c^{a} \in \mathbb{R}^{3}$ satisfying

$$
\mu>0, \quad \nu>0, \quad \beta_{*} \neq 0, \quad c^{a} \neq 0,
$$

there exists a solution $h$ to the conformal static field equations which admits a non-trivial conformal rescaling with conformal factor $\vartheta=\sqrt{\nu / u}$. The fields $h$ and $u$ are uniqely determined by the requirements that $m=2 \sqrt{\mu}$ is the ADM mass of the asymptotically flat static metric associated with $h$, the Ricci tensor of $h$ assumes in $i$-centered $h$-normal coordinates the value

$$
s_{a b}=\beta_{*}\left(c_{a} c_{b}-\frac{1}{3} h_{a b} c_{d} c^{d}\right) \neq 0 \quad \text { at } \quad i,
$$

and the positive function u satisfies

$$
u=\nu, \quad D_{a} u=c_{a} \quad \text { at } \quad i .
$$

Proof. The result will be obtained by solving simultaneously the conformal field equations for $h$ and the equations satisfied by $u$. Let $e_{a}, a=1,2,3$, denote an $h$-orthonomal frame and denote the 1 -forms dual to it by $\sigma^{a}$. The metric is then given by $h=h_{a b} \sigma^{a} \sigma^{b}$ with metric coefficients $h_{a b}=h\left(e_{a}, e_{b}\right)=-\delta_{a b}$. The connection coefficients, defined by $D_{e_{a}} e_{b}=\Gamma_{a}{ }^{c}{ }_{b} e_{c}$, satisfy $\Gamma_{a c b}=\Gamma_{a[c b]}$ with $\Gamma_{a c b}=h_{c d} \Gamma_{a}{ }^{d}{ }_{b}$. The connection form is then given by $\omega^{a}{ }_{b}=\Gamma_{c}{ }^{a}{ }_{b} \sigma^{c}$ so that $\omega_{a b}=\omega_{[a b]}$. This expansion in terms of the $\sigma^{a}$ will be used only later, when we describe the solution procedure in detail.

The equations will be written as differential system for the unknown

$$
\mathbf{U}=\left(\rho, \rho_{a}, s, u, u_{a}, t, \beta, \sigma^{a}, \omega^{a}{ }_{b}\right),
$$

where the first seven components denote (vector-valued) 0-forms and the last two components are 1-forms. Until we introduce coordinates below all indices should be understood as frame indices. Consider the differential forms

$$
\Lambda=d \rho-\rho_{a} \sigma^{a}, \quad \Gamma=d u-u_{a} \sigma^{a},
$$




$$
\begin{gathered}
\Sigma_{a}=d \rho_{a}-\rho_{c} \omega^{c}{ }_{a}-s h_{a b} \sigma^{b}+\rho(1-\rho) s_{a b} \sigma^{b}, \\
\Pi_{a}=d u_{a}-u_{c} \omega^{c}{ }_{a}-t h_{a b} \sigma^{b}+u(1-u) s_{a b} \sigma^{b}, \\
S=d s+(1-\rho) \rho^{a} s_{a b} \sigma^{b}, \quad T=d t+(1-u) u^{a} s_{a b} \sigma^{b}, \\
B=d \beta+\beta M_{a} \sigma^{a}, \\
\Theta^{a}=d \sigma^{a}+\omega^{a}{ }_{b} \wedge \sigma^{b}, \quad \Delta^{a}{ }_{b}=d \omega^{a}{ }_{b}+\omega^{a}{ }_{c} \wedge \omega^{c}{ }_{b}-\Omega^{a}{ }_{b},
\end{gathered}
$$

denoted collectively by

$$
\boldsymbol{\Psi}=\left(\Lambda, \Gamma, \Sigma_{a}, \Pi_{a}, S, T, B, \Theta^{a}, \Delta^{a}{ }_{b}\right),
$$

or by $\boldsymbol{\Psi}^{A}$ if we need to bring out relations involving different components. In the differential forms above and in the forms derived below we consider functions of the components of $\mathbf{U}$ which are given by

$$
\begin{gathered}
w=\frac{1-u}{1-\rho}, \quad w_{a}=\frac{1-u}{(1-\rho)^{2}} \rho_{a}-\frac{1}{1-\rho} u_{a}, \quad s_{a b}=\beta\left(w_{a} w_{b}-\frac{1}{3} h_{a b} w_{c} w^{c}\right) \\
M_{a}=Q w_{a}+\frac{1}{3}(1-u)(1-w) \beta w_{a}-\frac{1}{1-\rho} \rho_{a}, \quad Q=\frac{5\left(w s-t+\rho_{c} w^{c}\right)}{w_{e} w^{e}(1-\rho)} \\
\Omega_{b}^{a}=\frac{1}{2} R_{b c d}^{a} \sigma^{c} \wedge \sigma^{d}=\left(h^{a}{ }_{c} s_{b d}-h_{b c} s_{d}{ }^{a}\right) \sigma^{c} \wedge \sigma^{d} .
\end{gathered}
$$

The equations we need to solve read now

$$
\Psi=0 .
$$

The first two of these equations ensure that $\rho_{a}$ and $u_{a}$ represent the differentials of $\rho$ and $u$, the following five equations represent (2.2), (3.9), (2.9), (3.11), (4.9) and the remaining equations are the first and the second structural equation.

A lengthy but straight forward calculation shows that the differential forms comprised by $\boldsymbol{\Psi}$ satisfy the differential system

$$
\begin{gathered}
d \Lambda=-\Sigma_{a} \wedge \sigma^{a}-\rho_{a} \Theta^{a}, \\
d \Gamma=-\Pi_{a} \wedge \sigma^{a}-u_{a} \Theta^{a}, \\
d \Sigma_{a}=-\Sigma_{b} \wedge \omega^{b}{ }_{a}-\rho_{b} \Delta^{b}{ }_{a}-S \wedge \sigma_{a}-s \Theta_{a}+(1-2 \rho) \Lambda \wedge s_{a b} \sigma^{b}+\rho(1-\rho) s_{a b} \Theta^{b}+\rho(1-\rho) \mathcal{A}_{a}, \\
d \Pi_{a}=-\Pi_{b} \wedge \omega^{b}{ }_{a}-u_{b} \Delta^{b}{ }_{a}-T \wedge \sigma_{a}-t \Theta_{a}+(1-2 u) \Gamma \wedge s_{a b} \sigma^{b}+u(1-u) s_{a b} \Theta^{b}+u(1-u) \mathcal{A}_{a}, \\
d T=-\Lambda \wedge \rho^{a} s_{a b} \sigma^{b}+(1-\rho) \Sigma_{a} \wedge s^{a}{ }_{b} \sigma^{b}+(1-\rho) \rho^{a} s_{a b} \Theta^{b}+(1-\rho) \rho^{a} \mathcal{A}_{a}, \\
d S=-\Gamma \wedge u^{a} s_{a b} \sigma^{b}+(1-u) \Pi_{a} \wedge s^{a}{ }_{b} \sigma^{b}+(1-u) u^{a} s_{a b} \Theta^{b}+(1-u) u^{a} \mathcal{A}_{a},
\end{gathered}
$$




$$
\begin{gathered}
d B=B \wedge\left(M_{a} \sigma^{a}+\beta F w_{a} \sigma^{a}\right) \\
+\beta\left\{\frac{Q}{1-\rho} \Lambda+\left(\frac{5 \rho^{c}}{(1-\rho)^{3} w_{b} w^{b}}-\frac{2 Q w^{c}}{(1-\rho)^{2} w_{b} w^{b}}\right)\left(-\rho_{c} \Gamma+w \rho_{c} \Lambda+(1-\rho)\left(w_{c} \Lambda+w \Sigma_{c}-\Pi_{c}\right)\right)\right. \\
\left.+\frac{5}{(1-\rho)^{2} w_{b} w^{b}}\left(s w \Lambda-s \Gamma+(1-\rho)\left(w S-T+w^{c} \Sigma_{c}\right)\right)\right\} \wedge w_{a} \sigma^{a} \\
+\beta\left\{\frac{Q+\beta F}{(1-\rho)^{2}}\left(w \rho_{a} \Lambda-\rho_{a} \Gamma+(1-\rho)\left(w_{a} \Lambda+w \Sigma_{a}-\Pi_{a}\right)\right)-\frac{1}{3} \beta\left((1-2 w) \Gamma+w^{2} \Lambda\right) w_{a}\right. \\
\left.-\frac{\rho_{a}}{(1-\rho)^{2}} \Lambda-\frac{1}{1-\rho} \Sigma_{a}\right\} \wedge \sigma^{a}+\beta M_{a} \Theta^{a}, \\
d \Delta^{a}{ }_{b}=\Delta^{a}{ }_{c} \wedge \omega^{c}{ }_{b}-\omega^{a}{ }_{c} \wedge \Delta^{c}{ }_{b}{ }_{b}-\left(h^{a}{ }_{c}{ }^{a}{ }_{b b d}-h_{b c}{ }_{b} \wedge{ }^{b}{ }^{a}{ }^{b}\right)\left(\Theta^{c} \wedge \sigma^{d}{ }_{b} \wedge \Theta^{b},\right. \\
\left.\sigma^{c} \wedge \Theta^{d}\right)+\sigma^{a} \wedge \mathcal{A}_{b}-\mathcal{A}^{a} \wedge \sigma_{b},
\end{gathered}
$$

where

$$
\mathcal{A}_{a}=w_{a} B \wedge w_{b} \sigma^{b}-\frac{1}{3} w_{b} w^{b} B \wedge \sigma_{a}+\beta\left(\mathcal{B}_{a} \wedge w_{b} \sigma^{b}+w_{a} \mathcal{B}_{b} \wedge \sigma^{b}-\frac{2}{3} w^{b} \mathcal{B}_{b} \wedge \sigma_{a}\right),
$$

with

$$
\mathcal{B}_{a}=-\frac{\rho_{a}}{(1-\rho)^{2}} \Gamma+\left(\frac{w_{a}}{1-\rho}+\frac{w \rho_{a}}{(1-\rho)^{2}}\right) \Lambda+\frac{w}{1-\rho} \Sigma_{a}-\frac{1}{1-\rho} \Pi_{a} .
$$

In deriving the last equation there arises the expression

$\mathcal{N}^{a b}=-w_{d} w^{d} \sigma^{a} \wedge \rho_{c} \sigma^{c} \wedge \sigma^{b}+\rho_{c} w^{c} \sigma^{a} \wedge w_{d} \sigma^{d} \wedge \sigma^{b}+\sigma^{a} \wedge \rho_{c} \sigma^{c} \wedge w_{d} \sigma^{d} w^{b}-w^{a} \rho_{c} \sigma^{c} \wedge w_{d} \sigma^{d} \wedge \sigma^{b}$.

By writing $\sigma^{a} \wedge \sigma^{b} \wedge \sigma^{c}=\epsilon^{a b c} \mu_{h}$ with the volume form defined by $h$ on the right hand side and calculating $\mathcal{N}^{a b} \epsilon_{a b c}$, the field $\mathcal{N}^{a b}$ can be shown to vanish identically.

In short notation (the summation convention applying to all indices) the relations above take the form

$$
d \boldsymbol{\Psi}^{A}=f_{B c}^{A} \sigma^{c} \wedge \boldsymbol{\Psi}^{B}+f_{B b}^{A a} \omega^{b}{ }_{a} \wedge \boldsymbol{\Psi}^{B}+f_{B}^{A} \boldsymbol{\Psi}^{B},
$$

with functions $f_{B c}^{A}, f_{B b}^{A a}, f_{B}^{A}$ of the 0 -forms given by $\mathbf{U}$.

Because we assume that $D_{a} u(i)=c_{a} \neq 0$, all fields given by $\mathbf{U}$, in particular $\beta$ (with the meaning given to it earlier), can be assumed to be smooth near $i$. The solutions to the equations $\boldsymbol{\Psi}=0$ are obtained near $i$ as follows. Assume that $x^{a}$ denote $i$-centered, $h$-normal coordinates and $e_{a}$ an $h$-orthonormal normal frame centered at $i$ so that $e^{a}{ }_{b}=$ $<d x^{a}, e_{b}>=\delta^{a}{ }_{b}$ at $i$. Then $\hat{Y}=x^{a} \partial_{a}$ is smooth near $i$ and so that $Y=\frac{1}{|x|} \hat{Y}$ is for $x^{a} \neq 0$ the geodesic unit vector field tangent to the geodesics through $i$, which has direction dependent limits as $|x| \rightarrow 0$. Writing $\sigma^{a}=\sigma^{a}{ }_{b} d x^{b}$, we find the inner products

$$
i_{\hat{Y}} \sigma^{a}=\sigma_{b}^{a}(x) x^{b}=x^{a}, \quad i_{\hat{Y}} \omega_{b}^{a}=x^{c} \Gamma_{c}{ }^{a}{ }_{b}(x)=0 \quad \text { near } \quad x^{a}=0 .
$$


Consider now a radial geodesic $\gamma: \tau \rightarrow \tau x_{*}^{a}$ with some $x_{*}^{a}$ satisfying $\delta_{a b} x_{*}^{a} x_{*}^{b}=1$. The equation

$$
i_{Y} \Theta^{a}=0,
$$

implies then for the unknown $\hat{\sigma}^{a}{ }_{b}=\sigma^{a}{ }_{b}-\delta^{a}{ }_{b}$, which is required to be smooth, the initial condition

$$
\hat{\sigma}_{b}^{a} \rightarrow 0 \text { as } \tau \rightarrow 0
$$

and along $\gamma$ the ODE

$$
\frac{d}{d \tau} \hat{\sigma}_{b}^{a}+\frac{1}{\tau} \hat{\sigma}_{b}^{a}=\Gamma_{c}{ }^{a}{ }_{d} \hat{\sigma}^{c}{ }_{b} x_{*}^{d}+\Gamma_{b}{ }^{a}{ }_{d} x_{*}^{d},
$$

where we expressed the connection form in terms of the connection coefficients, which are considered now as unknowns. The equation

$$
i_{Y} \Delta^{a}{ }_{b}=0,
$$

similarly implies the initial condition and the ODE

$$
\begin{gathered}
\Gamma_{c}{ }^{a}{ }_{b} \rightarrow 0 \text { as } \tau \rightarrow 0, \\
\frac{d}{d \tau} \Gamma_{c}{ }^{a}{ }_{b}+\frac{1}{\tau}\left(\delta^{d}{ }_{c}-\hat{\sigma}^{d}{ }_{f} e^{f}{ }_{c}\right) \Gamma_{d}{ }^{a}{ }_{b}=-\Gamma_{d}{ }^{a}{ }_{b} \Gamma_{c}{ }^{d}{ }_{e} x_{*}^{e}+R^{a}{ }_{b d c} x_{*}^{d},
\end{gathered}
$$

where the frame coefficients $e^{a}{ }_{b}$ (by Cramer's rule rational functions of the $\sigma^{a}{ }_{b}$ ) satisfy

$$
\sigma^{a}{ }_{c} e^{c}{ }_{b}=\delta^{a}{ }_{b} \text { so that } \quad e^{a}{ }_{b}=\delta^{a}{ }_{b}-\hat{\sigma}^{a}{ }_{c} e^{c}{ }_{b}=\delta^{a}{ }_{b}+O(|\tau|) .
$$

The equations

$$
i_{Y} \Lambda=0, \quad i_{Y} \Gamma=0, \quad i_{Y} \Sigma_{a}=0, \quad i_{Y} \Pi_{a}=0, \quad i_{Y} S=0, \quad i_{Y} T=0, \quad i_{Y} B=0,
$$

imply regular ODE's along $\gamma$. The unknowns in these equations must satisfy the initial conditions

$$
\rho=0, \rho_{a}=0, s=-2 \mu, u=\nu>0, u_{a}=c_{a} \neq 0, t=\frac{c_{a} c^{a}}{2 \nu}, \beta=\beta_{*} \neq 0 \quad \text { at } \quad \tau=0 .
$$

There exists along $\gamma$ a unique solution $\mathbf{U}$ to the system consisting of (5.5), (5.7), and the ODEs implied by (5.8) which satisfies the conditions (5.3), (5.4), (5.6), (5.9). This solution is real analytic in the initial data (5.9) and $\tau x_{*}^{a}$. The fields given by $\mathbf{U}$ are in fact analytic in the coordinates $x^{a}$ and satify the equation $\boldsymbol{\Psi}=0$ near $x^{a}=0$.

This result follows from general properties of systems of ODE's. The only subtlety arises here from the singularity of equations (5.5), (5.7) at $\tau=0$. It is such that the left hand sides of these equations are of the form $\dot{x}+\tau^{-1} A x$ with a matrix $A$ which approaches a diagonal matrix with positive entries as $\tau \rightarrow 0$. The existence of an unique analytic solution to the complete system then follows immediately with the methods used in [5] where ODE's of the same type have been discussed.

Once the solution $\mathbf{U}$ has been obtained, equations (5.5), (5.7) imply a system of ODE's for $x_{*}^{a} \hat{\sigma}^{a}{ }_{b}$ and $x_{*}^{a} \Gamma_{a}{ }^{b}{ }_{c}$ along $\gamma$ which allows one to concluce that these quantities vanish 
everywhere so that the relations (5.3) are indeed satisfied. Because $i_{Y} \boldsymbol{\Psi} \rightarrow 0$ as $x^{a} \rightarrow 0$ along any geodesic passing through $x^{a}=0$ it follows that $\boldsymbol{\Psi}=0$ at $x^{a}=0$. From (5.2), (5.3), and $i_{Y} \boldsymbol{\Psi}=0$ one obtains

$$
\mathcal{L}_{Y} \boldsymbol{\Psi}^{A}=\left(d \circ i_{Y}+i_{Y} \circ d\right) \boldsymbol{\Psi}^{A}=i_{Y} d \boldsymbol{\Psi}^{A}=f_{B c}^{A} \frac{x^{c}}{|x|} \boldsymbol{\Psi}^{B} .
$$

This equation implies for $|x| \neq 0$ along each geodesic $\gamma$ passing through $x^{a}=0$ a linear homogeneous system of ODE's for the coefficient functions defining the forms given by $\boldsymbol{\Psi}$. It behaves regularly on $\gamma$ as $|x| \rightarrow 0$. Since $\boldsymbol{\Psi}$ vanishes at $x^{a}=0$, the asserted result follows.

In counting the free parameters in lemma 5.2 the vector $c^{a}$ should be taken into account only in terms of the number $c=\sqrt{c_{a} c^{a}}>0$ because of the freedom to rotate the normal coordinates around their origin. Ignoring the parameter $\mu$, which can be changed by trivial rescalings, it follows the that solutions depend on three parameters.

\section{Concluding remarks}

The statements of theorem 1.1 represent an extract from the results proven in the lemmas and the subsequent remarks. The latter provide much more information on the various structures. This will become important in a sequel to this article in which the properties of the solutions whose existence has been obtained in theorem 1.1 will be discussed in some detail.

\section{References}

[1] R. Beig. Conformal properties of static spacetimes. Class. Quantum Grav. 8 (1991) $263-271$.

[2] R. Beig, W. Simon. Proof of a multipole conjecture due to Geroch. Comm. Math. Phys. 78 (1980) $75-82$.

[3] H. W. Brinkmann. Einstein spaces which are mapped conformally on each other. Math. Ann. 94 (1925) 119 - 145.

[4] H. Friedrich. Smoothness at null infinity and the structure of initial data. In: P. T. Chruściel, H. Friedrich (eds.): The Einstein equations and the large scale behaviour of gravitational fields. Birkhäuser, Basel, 2004.

[5] H. Friedrich. Static vacuum solutions from convergent null data expansions at spacelike infinity. Ann. Henri Poincare 8 (2007) 817 - 884.

[6] L. Hörmander. The analysis of linear partial differential operators I. Springer, Berlin 1983.

[7] D. Kennefick, N. O'Murchadha. Weakly decaying asymptotically flat static and stationary solutions to the Einstein equations. Class. Quantum. Grav. 12 (1995) 149-158. 
[8] H. Stephani, D. Kramer, M. MacCallum, C. Hoenselaers, E. Herlt. Exact solutions of Einstein's field equations. Cambridge University Press, Cambridge, 2003.

[9] K. Yano. The theory of Lie derivatives and its applications. Amsterdam, North Holland, 1957. 